\title{
Revisões Sistemáticas e Metanálises na Reumatologia
}

\section{Systematic Review and Meta-analyses Rheumatology}

\author{
Rachel Riera ${ }^{(1)}$, Mirhelen Mendes de $\mathrm{Abreu}^{(2)}$, Rozana Mesquita Ciconelli( ${ }^{(3)}$
}

\section{RESUMO}

O conhecimento científico tem crescido exponencialmente, fazendo com que médicos e tomadores de decisão estejam inundados de informações, necessitando integrá-las de modo consistente. A fim de otimizar as tomadas de decisão, os estudos de revisão, caracterizados como revisão sistemática e metanálise, estabelecem tais consistências e avaliam se seus resultados podem ser extrapolados para a população em geral ou a um paciente em particular. Este artigo propõe a revisão de conceitos gerais envolvendo tais estudos, enfatizando suas propriedades metodológicas para usá-las na prática clínica.

Palavras-chave: revisão sistemática, metanálise, tomada de decisão médica, conceitos.

\section{INTRODUÇÃO}

Considerando o crescente número de informações disponíveis na área da saúde e a evidente dificuldade do profissional desse setor em manter-se atualizado, estudos de revisão têm sido úteis na aquisição de novos conhecimentos. De modo particular, as revisões sistemáticas e as metanálises se tornaram a melhor fonte de evidência na elaboração de estratégias de saúde pública, bem como na tomada de decisão clínica individual ${ }^{(1)}$. As revisões narrativas são úteis para responder questões sobre história de um evento e para comparar ou integrar diferentes áreas de pesquisa. No entanto, não fornecem respostas quantitativas para questões de tratamento e prevenção $(\text { Tabela } 1)^{(2)}$.

\begin{abstract}
Science knowledge is growing exponentially. Doctors and policy makers are inundated with amounts of information, needing systematic reviews to efficiently integrate existing information and provide data for rational decision-making. To improve the medical decision making in a better conditions, systematic reviews, including meta-analyses, establish whether scientific findings efficiently are consistent and can be generalized across populations or a patient in particular way. This paper aims to update the concepts of these kind of studies, emphasizing their methodological principles to be used in the clinical practice.
\end{abstract}

Keywords: systematic reviews, meta-analyses, medical decision making, concepts.

A revisão sistemática é um estudo retrospectivo secundário que identifica, seleciona e avalia, criticamente, estudos primários (coorte, acurácia, ensaios clínicos, etc), permitindo a somatória dos seus resultados e transformando informação em conhecimento ${ }^{(3)}$. Proporciona uma visão geral clara e reprodutível dos estudos primários, avalia o benefício ou não de uma intervenção e identifica os erros e acertos dos estudos, possibilitando que um novo estudo seja planejado de forma mais adequada. Ela permite, muitas vezes, explicar as diferenças encontradas entre estudos primários que investigam a mesma questão ${ }^{(2)}$. Dependendo da pergunta que se pretende responder, a revisão sistemática pode incluir ensaios clínicos randomizados, estudos de acurácia, estudos coortes ou qualquer outro tipo de desenho ${ }^{(4)}$.

Disciplina de Reumatologia da Escola Paulista de Medicina da Universidade Federal de São Paulo (UNIFESP). Recebido em 17/02/05. Aprovado, após revisão, em 10/05/05.

1. Especializanda da Disciplina de Reumatologia da Escola Paulista de Medicina da UNIFESP.

2. Pós-graduanda da Disciplina de Reumatologia da Escola Paulista de Medicina da UNIFESP.

3. Professora da Disciplina de Reumatologia da Escola Paulista de Medicina da UNIFESP e Membro do Projeto Pronuclear da Sociedade Brasileira de Reumatologia (SBR).

Endereço para correspondência: Rachel Riera, Rua José de Magalhães, 373/1202B, Vila Clementino, São Paulo, CEP 04026- 090, SP, Brasil,

e-mail: rachel.dmed@unifesp.epm.br 
TABELA 1

Tipos de Revisão da Literatura ${ }^{(2)}$

\begin{tabular}{|c|c|c|}
\hline & Revisão narrativa & Revisão sistemática \\
\hline Questão & ampla & específica \\
\hline Fonte & $\begin{array}{l}\text { freqüentemente não- } \\
\text { especificada, com grande } \\
\text { risco de viés }\end{array}$ & $\begin{array}{l}\text { fontes abrangentes, } \\
\text { estratégia de busca } \\
\text { definida previamente }\end{array}$ \\
\hline $\begin{array}{l}\text { Seleção dos } \\
\text { estudos }\end{array}$ & $\begin{array}{l}\text { freqüentemente não- } \\
\text { especificada, com grande } \\
\text { risco de viés }\end{array}$ & $\begin{array}{c}\text { baseada em critérios } \\
\text { pré-definidos e aplicados } \\
\text { uniformemente }\end{array}$ \\
\hline $\begin{array}{l}\text { Avaliação dos } \\
\text { estudos }\end{array}$ & variável & criteriosa e reprodutível \\
\hline Sintese & qualitativa & $\begin{array}{l}\text { quantitativa com método } \\
\text { estatístico (metanálise) }\end{array}$ \\
\hline Inferências & $\begin{array}{l}\text { às vezes baseada em } \\
\text { resultados de pesquisa } \\
\text { clinica }\end{array}$ & $\begin{array}{c}\text { freqüentemente baseada } \\
\text { em resultados de pesquisa } \\
\text { clínica }\end{array}$ \\
\hline
\end{tabular}

\section{VANTAGENS DA REVISÃo SISTEMÁtICA}

A revisão sistemática, aceita como tese de doutorado e livre-docência nas melhores escolas de medicina, é validada como método de pesquisa nas mais rigorosas revistas médicas e apresenta numerosas vantagens ${ }^{(5-16)}$ :

a) utiliza um método científico que pode ser reproduzido na prática clínica;

b) evita a duplicação desnecessária de esforços, uma vez que quando se completa a revisão ela não precisa ser repetida por outro grupo;

c) pode ser atualizada periodicamente, sem maiores esforços, por meio da busca de novos ensaios clínicos publicados, que, se forem de boa qualidade, serão incluídos na metanálise;

d) reduz controvérsias na literatura, uma vez que não resulta do número de estudos favoráveis a determinada intervenção, mas da soma de todos os casos estudados;

e) antecipa o resultado de grandes ensaios clínicos que ainda esperam para serem realizados, devido às dificuldades técnicas e/ou financeiras;

f) pode detectar tratamentos inadequados em estágios mais iniciais de seu uso, portanto, salvando um grande número de pacientes de efeitos adversos de tratamentos desnecessários;

g) aumenta a acurácia dos resultados, diminuindo o intervalo de confiança e aumentando o número da amostra;

h) direciona futuros estudos para áreas deficientes em evidências;

i) economiza recursos em pesquisa clínica e assistência médica;

j) auxilia decisões para políticas de saúde; k) exige trabalho em equipe, cooperação, e "negociação" de opiniões, o que nem sempre é fácil, embora seja uma habilidade fundamental a ser desenvolvida pelos profissionais da área da saúde.

DESVANTAGENS $(1,2,17)$

a) Como qualquer pesquisa científica de boa qualidade, uma revisão sistemática consome tempo, podendo demorar de três meses a um ano para ser concluída;

b) exige dedicação, grande esforço intelectual (formular a pergunta, desenvolver a estratégia de pesquisa, comparar os trabalhos e interpretar os dados);

c) não consegue melhorar diretamente a qualidade dos estudos que compõem a revisão sistemática. Pode apenas recomendar que os novos estudos não cometam os mesmos erros, promovendo de modo indireto a melhora da qualidade dos ensaios clínicos subseqüentes;

d) envolve, pelo menos, dois profissionais para avaliar os ensaios clínicos, sendo praticamente impossível fazer uma revisão sistemática sozinho.

\section{O PAPEL DA REVISÃo SISTEMÁtICA NA PRÁTICA CLÍNICA}

É importante ressaltar que a revisão sistemática é apenas uma parte do processo que resulta na decisão clínica. Dessa forma, apenas o resultado de uma revisão sistemática ainda não é suficiente. A conduta clínica deve ser baseada no resultado das pesquisas, na experiência do profissional, no contexto do atendimento (ambiente e particularidades dos doentes), na situação econômica e também na opinião do próprio paciente (Figura 1$)^{(18,19)}$. Assim, considerando todos esses fatores, teremos o que

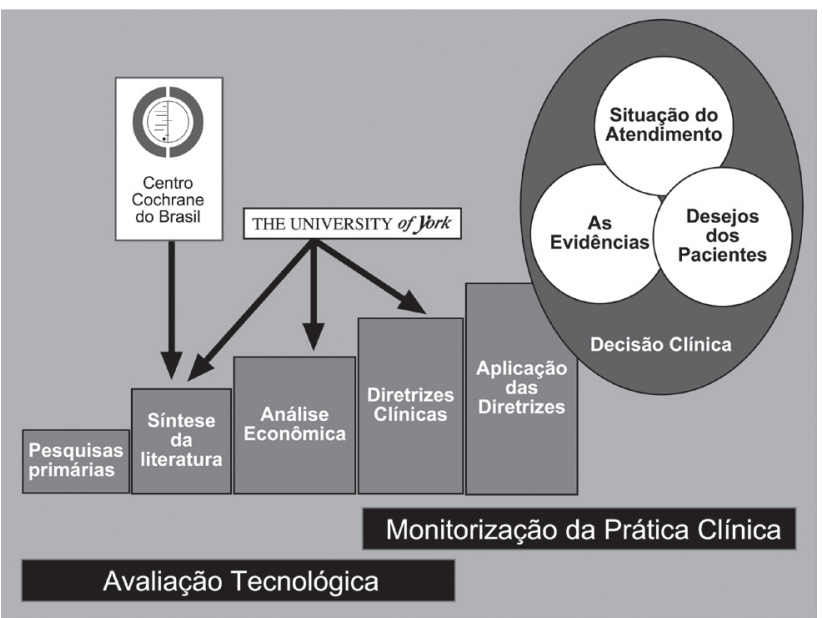

Figura 1 - Bases da Decisão Clínica ${ }^{(18)}$. 
se chama de diretriz clínica ou guideline. ${ }^{(20)}$

Deve ser lembrado, também, que as características locais das doenças e as dificuldades para implantação de intervenções adequadas variam muito conforme cada região ou país, e a atenção para estes itens locais é que permitirá a aplicação dos resultados da pesquisa.

No que diz respeito à tomada de decisão para um único indivíduo, os resultados das revisões sistemáticas devem ser integrados à experiência do profissional de saúde, ao conhecimento prévio do doente em relação à sua condição (principalmente no caso de problemas crônicos ou recorrentes), aos tratamentos disponíveis e à resposta a esses tratamentos $^{(18)}$.

As considerações anteriores também são úteis na avaliação da relevância das revisões sistemáticas na tomada de decisão em saúde pública ${ }^{(21,22)}$. Porém, dificilmente os resultados de uma revisão sistemática têm implicações universais. Além disso, a aplicação de recomendações e prescrições universais nem sempre são sábias nem funcionais.

\section{METANÁLISE}

Quando aplicamos um método estatístico à somatória dos resultados dos estudos primários, obtemos uma síntese quantitativa chamada metanálise ${ }^{(3)}$. A primeira metanálise foi publicada em 1904, no British Medical Journal e, em 1955, a primeira metanálise envolvendo uma questão clínica foi publicada no JAMA ${ }^{(10)}$. Porém, o termo metanálise surgiu apenas em 1976, em artigo da revista Educational Research ${ }^{(10)}$. As revisões sistemáticas e metanálises se destacaram na área da saúde no final dos anos 80, com a publicação do livro Effective Care During Pregnancy and Childbirth, considerado um marco na história da obstetrícia moderna ${ }^{(10)}$.

Em 1992, uma publicação de Iain Chalmers no BMJ enumerou os objetivos de uma cooperativa dedicada à facilitar e disseminar revisões sistemáticas ${ }^{(23)}$. As estratégias de busca de ensaios clínicos aleatórios em bases de dados foram definidas em 1994, em outra publicação do BMJ ${ }^{(24)}$.

Também no ano de 1992 foram desenvolvidas as duas primeiras teses que consistiam em revisões sistemáticas com metanálises, uma na Inglaterra e outra no Brasil ${ }^{(10)}$. Em Oxford, Alejandro Jadad defendeu sua tese de doutorado e, em São Paulo, Jair de Jesus Mari defendeu sua tese de livre-docência na Escola Paulista de Medicina da Universidade Federal de São Paulo (UNIFESP) ${ }^{(25)}$. Em 1997, também na UNIFESP, foi defendida a primeira tese de doutoramento compreendendo uma revisão sistemática com metanálise ${ }^{(26)}$.

\section{A COLABORAÇÃO COCHRANE}

Em 1992 (Oxford - Reino Unido), o Dr. Iain Chalmers criou a Colaboração Cochrane, cujos objetivos são realizar, auxiliar e disseminar revisões sistemáticas de intervenções em saúde.

A Colaboração Cochrane possui centros em vários países do primeiro mundo, unindo força e competência para que as decisões médicas sejam baseadas nas melhores evidências científicas disponíveis. Em 31 de outubro de 1997, foi fundado o Centro Cochrane do Brasil, na UNIFESP (http://www.centrocochranedobrasil.com.br), que é o primeiro a ser credenciado em um país em desenvolvimento. Todo o trabalho da Colaboração Cochrane tem sido voluntário e sem fins lucrativos. A Colaboração Cochrane e os Centros Cochrane realizam revisões sistemáticas, auxiliam os interessados a realizálas e divulgam resultados de revisões sistemáticas. Em virtude do grande impacto provocado tanto na prática médica, quanto nas políticas de saúde e nas definições de prioridades em pesquisas clínicas, o trabalho da Colaboração Cochrane tem sido considerado o correspondente clínico do Projeto Genoma ${ }^{(10)}$.

\section{BIBLIOTECA COCHRANE}

Uma das formas mais acessíveis de divulgação de revisões sistemáticas é a Cochrane Library, publicação eletrônica editada pela UpDate Software e que contém revisões sistemáticas prontas, revisões em andamento (protocolos) e um extenso banco de dados de ensaios clínicos sobre diversos assuntos, atualizados a cada três meses que estão disponíveis gratuitamente (http://bireme.br/cochrane) aos profissionais preocupados em encontrar evidências cientificas que sustentem suas condutas.

A primeira edição de 2005 da Biblioteca Cochrane contém 2.249 revisões sistemáticas completas e 1.539 protocolos de revisões, 435.786 resumos de ensaios clínicos randomizados, além das bases de dados de estudos de efetividade e economia em saúde, o que reflete a dedicação de milhares de pessoas envolvidas com a Colaboração Cochrane no mundo todo.

Alguns grupos temáticos da Colaboração Cochrane são de maior interesse para os profissionais da área reumatológica como o Grupo Musculoesquelético, o Grupo Neuromuscular, o Grupo de lesões musculoesqueléticas e o Back Group (Tabela 2).

Em resumo, podemos dizer que uma revisão sistemática de qualidade faz parte do elo que une a evidência científica de qualidade e a boa prática clínica, ou seja, integra a medicina baseada em evidências ${ }^{(1)}$. 
TABELA 2

Revisões Sistemáticas em Reumatologia da Colaboração Cochrane

\begin{tabular}{|c|c|}
\hline Grupo Temático & Tema de interesse na reumatologia \\
\hline Musculoesquelético & $\begin{array}{l}\text { gota (1), lúpus (2), osteoartrose (37), osteoporose (21), artrite reumatóide juvenil (2), artrite reumatóide (43), osteonecrose (1), fibromialgia (6), } \\
\text { vasculite (6), espondiloartropatias (8), reumatismo partes moles (26), esclerose sistêmica (5), doença de Paget (1), doença de Legg-Perthes (1) }\end{array}$ \\
\hline $\begin{array}{l}\text { Lesões } \\
\text { musculoesqueléticas }\end{array}$ & $\begin{array}{l}\text { partes moles esqueleto apendicular (8), reabilitação (5), lesão tendão mãos (2), ligamento joelhos (8), menisco (5), síndrome patelo-femora } \\
\text { (3), tendão calcâneo (2), lesões partes moles de membros inferiores (3), hálux valgo (1), ossificação heterotópica (1), dor região plantar (1) }\end{array}$ \\
\hline Neuromuscular & $\begin{array}{l}\text { miopatia inflamatória (5), miopatia metabólica (3), síndrome do túnel carpo (5), meralgia parestésica (1), neuroma de Morton (1), encurtamento } \\
\text { tendão calcâneo (4), pé caído (2) }\end{array}$ \\
\hline Back Group & $\begin{array}{l}\text { radiculopatia cervical (1), lesão cervical em chicote (10, cervicalgia mecânica (5), hérnia lombar discal (2), lombociatalgia (2), ciatalgia (2) } \\
\text { espondilose lombar (1), lombalgia (23) }\end{array}$ \\
\hline Vascular & doença de Kawasaki (3), fenômeno de Raynaud (1) \\
\hline Renal & nefrite lúpica (1), vasculite renal/ Goodpasture (1) \\
\hline Dermatologia & lúpus eritematoso discóide \\
\hline Vias aéreas & hipertensão pulmonar (5), doença intersticial pulmonar (9) \\
\hline Doenças genéticas & osteoporose por uso de corticoesteróides (1), osteogenese imperfecta (1), hipofosfatemia ligada ao X (1) \\
\hline Hematologia & bisfosfonatos no mieloma (1) \\
\hline Hepatobiliar & cirrose biliar primária (7) \\
\hline Cuidados paliativos & metástase óssea (4), distrofia simpático reflexa (1) \\
\hline
\end{tabular}

( ): Número de revisões disponíveis por tema.

\section{REFERÊNCIAS}

1. Atallah NA: Revisões sistemáticas da literatura e metanálise. Diagnóstico \& Tratamento 2: 12-5, 1997.

2. Cook DJ, Mulrow CD, Haynes B: Systematic reviews: synthesis of the best evidence for clinical decisions. Ann Intern Med 126: 376-380, 1997.

3. Clarke M, Oxman AD, editor(s): Cochrane Reviewer's Handbook 4.2.0 [updated April 2004]. The Cochrane Library. Oxford: Updated software 2: 2004.

4. Cook DJ, Mulrow CD, DavidoffF: Systematic reviews: critical links in the great chain of evidence. Ann Intern Med 126: 380-91, 1997.

5. Light RJ, Pillemer DB: Summing up: the science of reviewing research. Cambridge: MA: Harvard University Press, 1984.

6. Dickersin K, Berlin JA: Meta-analysis: state-of-the-science. Epidemiol Rev 14: 154-76, 1992.

7. Bossel JP, Blanchard J, Panak E, Peyrieux JC, Sacks H: Considerations for the meta-analysis of randomized clinical trials. CoBMJ 09: 597-599, 1994.

8. Cook DJ, Greengold NL, Ellrodt AG, Weingarten SR: The relation between systematic review and practice guidelines. Ann Intern Med 127: 210-6, 1997.

9. Mulrow CD: Systematic Reviews: Rationale for systematic reviews. Controlled Clin Trials 10: 254-81, 1989.

10. Castro AA, Saconato H, Guidugli F, Clark OAC: Curso de revisão sistemática e metanálise [on line]. São Paulo: LEDDIS/UNIFESP, 2002. Disponível em: URL: http://www. virtual.epm.br/cursos/metanalise

11. Antman EM, Lau J, Kupelnick B, Mosteller F, Chalmers TC: A comparison of results of meta-analyses of randomized control trials and recommendations of clinical experts. JAMA 268: 240-8, 1992.

12. Gelber RD, Goldhirsch A: Meta-analysis: the fashion of summing-up evidence. Ann Oncol 2: 461-8, 1991.
13. Mulrow CD: The medical review article: state of the science. Ann Intern Med 106:485-8, 1987.

14. Oxman AD, Guyatt GH: Guidelines for reading literature reviews. Can Med Assoc J 138: 697-703, 1988.

15. Chalmers I, Altman DG, eds: Systematic reviews. London: BMJ Publishing Group, 1995.

16. Chalmers I, Enkin M, Keirse MJNC, editors. Effective care in pregnancy and childbirth. Oxford: Oxford University Press, 1989.

17. Naylor CD: Grey zones of clinical practice: some limits to evidence-based medicine. Lancet 345: 840-2, 1995.

18. Haynes B, Haines A: Barriers and bridges to evidence based clinical practice. BMJ 25, 317: 273-276, 1998.

19. McAlister FA, Clark HD, van Walraven C, et al: The medical review article revisited: has the science improved? Ann Intern Med 131: 947-51, 1999.

20. Greenhalgh T: How to read a paper: papers that summarize other papers (systematic reviews and meta-analyses). BMJ 315: 672-5,1997.

21. Hunt DL, McKibbon KA: Locating and appraising systematic reviews. Ann Intern Med 126: 532-8, 1997.

22. Lau J, Joannidis JPA, Schmid CH: Quantitative synthesis in systematic reviews. Ann Intern Med 127: 820-6, 1997.

23. Chalmers I, Dickersin K, Chalmers TC: Getting to grips with Archie Cochrane's agenda. BMJ 3, 305: 786-8, 1992.

24. Scherer KDR, Lefebvre C: Systematic Reviews: Identifying relevant studies for systematic reviews. BMJ 309: 1286-291, 1994.

25. Mari JJ: Intervenções familiares e recaídas na esquizofrenia: metanálise dos resultados de pesquisas. Tese (Livre Docência). Universidade de São Paulo. São Paulo, 1994.

26. Soares KVS: Discinesia tardia induzida por neurolétricos: metanálise dos ensaios clínicos controlados. Tese (Doutorado). Universidade Federal de São Paulo. São Paulo, 1997. 